STUDI

FRANCESI

\section{Studi Francesi}

Rivista quadrimestrale fondata da Franco Simone

185 (LXII | II) | 2018

OCTAVE MIRBEAU: UNE CONSCIENCE AU TOURNANT

DU SIEECLE - sous la direction de Ida Merello

\title{
STEFANO RESCONI, Il canzoniere trobadorico U. Fonti, canone, stratigrafia linguistica
}

Andrea Giraudo

\section{(2) OpenEdition \\ Journals}

\section{Édition électronique}

URL : http://journals.openedition.org/studifrancesi/13185

DOI : $10.4000 /$ studifrancesi. 13185

ISSN : 2421-5856

Éditeur

Rosenberg \& Sellier

\section{Édition imprimée}

Date de publication : 1 août 2018

Pagination : 299-300

ISSN : 0039-2944

\section{Référence électronique}

Andrea Giraudo, « STEFANO RESCONI, // canzoniere trobadorico U. Fonti, canone, stratigrafia linguistica », Studi Francesi [En ligne], 185 (LXII | II) | 2018, mis en ligne le 01 septembre 2018, consulté le 05 janvier 2021. URL : http://journals.openedition.org/studifrancesi/13185;DOI : https://doi.org/10.4000/ studifrancesi. 13185

Ce document a été généré automatiquement le 5 janvier 2021.

\section{(c)}

Studi Francesi è distribuita con Licenza Creative Commons Attribuzione - Non commerciale - Non opere derivate 4.0 Internazionale. 


\title{
STEFANO RESCONI, Il canzoniere trobadorico U. Fonti, canone, stratigrafia linguistica
}

\author{
Andrea Giraudo
}

\section{RÉFÉRENCE}

STEFANO RESCONI, Il canzoniere trobadorico U. Fonti, canone, stratigrafia linguistica, Firenze, Edizioni del Galluzzo per la Fondazione Ezio Franceschini, 2014, «Corpus des Troubadours, 4. Études, 3», XII-382 pp. [con cd-rom].

1 Il volume di Stefano Resconi, che nasce come rielaborazione della tesi di dottorato, si colloca all'interno del filone di studi noto come "filologia dei canzonieri" ed è un'analisi complessiva del manoscritto trobadorico U (Firenze, Biblioteca Medicea Laurenziana, Plut. 41.43), contenente, tra gli altri, testi di importanti trovatori come Guiraut de Bornelh, Arnaut Daniel, Folquet de Marselha, Bernart de Ventadorn, Raimbaut de Vaqueiras, Peire Vidal, Raimbaut d'Aurenga e Bertran de Born. I principali obiettivi della ricerca, ossia la discussione della collocazione di U all'interno della tradizione manoscritta trobadorica e il luogo di compilazione del codice, sono perseguiti tramite un'ampia e articolata indagine di quattro aspetti, fortemente interrelati, che si riflettono nella scansione del volume: materialità, fonti, stratigrafia linguistica e struttura interna del canzoniere.

2 Il primo capitolo («Descrizione materiale del codice»), oltre al profilo paleografico e codicologico, ospita una prima ipotesi di collocazione geografica e storica del manoscritto, che risulterebbe compilato in Toscana (o nelle vicinanze) alla fine del XIII secolo. Il secondo capitolo («Studio delle fonti») esordisce con una panoramica degli studi sulla collocazione di $\mathrm{U}$ all'interno della tradizione, passando in rassegna le osservazioni di Gustav Gröber e di Salvatore Santangelo per giungere infine all'ipotesi avalliana della "terza tradizione", cui U dovrebbe appartenere e sulla cui esistenza sono 
di recente state avanzate alcune riserve. In quest'ottica, il seguito dell'analisi di Resconi consiste in una verifica stemmatica di tutti i componimenti presenti nel canzoniere. La disamina permette all'autore di riconoscere nel collettore $y$ di Avalle la fonte principale dei materiali poi confluiti in $\mathrm{U}$; risulta inoltre confermata la gemellarità di $\mathrm{U}$ e del canzoniere $c$, derivati da un antecedente comune $c^{1}$, e la consistenza della fonte $v^{2}$ comune a $\mathrm{U}$ e V${ }^{2}$; come risultato ulteriore, i dati esposti suggerirebbero «la sostanziale binarietà del sistema della tradizione manoscritta trobadorica» (p. 183).

Il terzo capitolo («Stratigrafia linguistica») si propone la localizzazione del manoscritto e l'individuazione di costanti della sua lingua. L'analisi è suddivisa in cinque sezioni: italianismi generici, settentrionalismi, tratti centrali e toscanismi, fenomeni peculiari o dubbi, elementi significativi del "sistema primario" (ossia la lingua d'oc) e della sua “immagine" propria del copista. L'analisi rivela un certo numero di tratti spiegabili con l'influsso di varietà italiane settentrionali (indicativo della circolazione dei materiali nell'Italia del Nord), ma soprattutto una presenza più frequente e sistematica di tratti che rimandano alla Toscana, in armonia con quanto già avanzato nel primo capitolo. In particolare, alcuni fenomeni maggiormente localizzanti porterebbero a Firenze e alla Toscana occidentale. Resconi propone, con cautela, una compilazione toscana centrale o fiorentina del codice, ipotizzando anche un passaggio per l'area pisano-lucchese dei materiali poi confluiti in $\mathrm{U}$; a questa regione rimanderebbero, oltre che le tracce linguistiche, anche alcune considerazioni sulla storia interna del canzoniere.

4 Il quarto capitolo («Struttura, canone, storia interna») propone dapprima l'analisi strutturale di $\mathrm{U}$, da cui si evince una sostanziale bipartizione del manoscritto, nonostante l'assenza di suddivisioni interne esplicite. La prima macrosezione "strutturata" secondo la definizione di Resconi, p. 270, e nettamente maggioritaria risponde a un criterio di tipo estetico invece che storico-cronologico ed è suddivisa in sezioni d'autore, disposte in ordine decrescente a seconda del valore attribuito a ciascuno, con la posizione liminare riservata a Guiraut de Bornelh. Questa macrosezione può essere definita come un'antologia "classica", nella misura in cui si rileva un interesse quasi esclusivo per trovatori nati e attivi in regioni d'oc tra la fine del XII e l'inizio del XIII secolo ed è contemplato soltanto un genere lirico, la canso. In questo quadro, si sottolinea la completa assenza di trovatori italiani e la scarsezza di quelli nati in Occitania, ma con carriera in parte italiana; tra questi, la preferenza è accordata ad autori attivi nelle corti nord-occidentali. La macrosezione "non strutturata", quasi a voler integrare la precedente, ospita invece sirventesi, testi dialogici, unica, trovatori italiani, cansos tradizionali - ma estranee alle coordinate geocronologiche della prima macrosezione - e altre in cui la fin'amor è declinata in modo peculiare; essa è inoltre caratterizzata anche da una piccola traccia di ordinamento alfabetico. Resconi passa poi a indagare le scelte di $\mathrm{U}$ in rapporto al suo presumibile pubblico, ossia quello toscano a cavallo tra Duecento e Trecento. Per quanto riguarda la prima macrosezione, il canone rappresenta - per genere lirico prescelto e coordinate autoriali e temporali - il retroterra su cui si era modulata l'esperienza siciliana, risultando dunque già attardato nella Toscana dell'epoca. Resconi sottolinea tuttavia la sovrapponibilità tra il canone trobadorico del De vulgari eloquentia e l'antologia "aulica" di U, la quale costituirebbe, insieme alla poesia siciliana, «gli unici modelli poetici ritenuti universalmente dotati di alto valore in un contesto di fortissimo fermento e discussione metapoetici» (p. 287), modelli che per Dante fungono da pietra di paragone per la poesia coeva «se intende sostenerne il pregio rispetto alle 'correnti' poetiche rivali» (ibid.). Il canone di $\mathrm{U}$ acquisterebbe dunque valore non solo come 
modello di stile, ma anche come «supporto per una critica talvolta militante» (ibid.), a prescindere da interessi puramente antiquari e con l'ovvia, ma tuttavia opportunamente esplicitata, avvertenza di non considerare $U$ «uno se non addirittura 'il' canzoniere provenzale che sta alla base della formazione trobadorica della prima maturità dantesca» (p. 289). Il quadro è rafforzato e precisato dalla possibilità di individuare altre forme della ricezione toscana della lirica trobadorica tra Due e Trecento, come il fatto che in U si trovano molti dei testi - anche rari - che i poeti italiani attivi in quest'area traducono o riecheggiano. In questa prospettiva "ricezionale" la macrosezione non strutturata, il cui scarto maggiore rispetto alla prima parte di U sta nella presenza di sirventesi, sarebbe forse da interpretare, con Resconi, come un tentativo "non invasivo" di integrazione di un genere, ossia la poesia politica, ben attestato in Toscana ma assente nell'esperienza siciliana; d'altro canto la posizione periferica all'interno del canzoniere potrebbe suggerire che queste aggiunte, nel momento stesso in cui completano l'antologia, siano paradossalmente sorpassate rispetto al modello aureo - e sempre attuale - della canso, in ragione della differenza di ispirazione conseguente al diverso scenario storico-politico. L'analisi di Resconi prosegue poi considerando la trascrizione "verso per verso" che, non essendo in questo caso accompagnata da notazione musicale, potrebbe essere indizio della volontà di porre più chiaramente in evidenza la struttura metrica, nell'ottica di una ricezione libresca della poesia trobadorica. All'interno di questa riflessione, un certo spazio viene dedicato al particolare trattamento dei versi brevi, soprattutto quando la rima è irrelata all'interno della cobla, rilevando che U tende ad accorparli a quelli vicini per formare unità metriche più estese, nell'ottica di una generale «conversione dei versi brevi provenzali a misure più prossime all'uso tanto dei trovatori attivi in Italia settentrionale nel corso del Duecento quanto alla poesia toscana» (p. 302). Dopo una sezione sulle attribuzioni erronee, discusse e omesse, il lavoro di Resconi si conclude con alcune considerazioni di storia interna, basandosi sull'analisi di due componimenti, l'unica tenso di U e un componimento del genovese Lanfranc Cigala. Entrambi i testi, databili l'uno al 1238 e l'altro al 1245 e disposti a brevissima distanza nella seconda parte del canzoniere, sono collocabili nel quadro dei rapporti tra Federico II e il marchese Bonifacio II del Monferrato, cui riservano critiche più o meno velate. Questo dato, unito alle considerazioni di affinità tra i due componimenti anche a livello di tradizione, permette a Resconi di individuare una «tradizione estremamente residuale e periferica» (p. 321), proponendo al contempo di identificare «un ambiente nel quale devono verosimilmente essere circolati alcuni dei materiali che hanno poi dato vita al canzoniere [...]: si tratta con tutta probabilità di un ambito ostile a Bonifacio, forse la stessa Genova» (ibid.). Resconi prosegue, appoggiandosi ad analoghe considerazioni di storia interna dell'altro canzoniere fortemente legato alla Toscana, $\mathrm{P}$, sostenendo che «perlomeno in questi casi, il passaggio di materiali trobadorici dalla pianura padana verso la Toscana sia avvenuto nella seconda metà del Duecento [...] grazie all'intermediazione delle corti liguro-piemontesi»(p. 322). Le tracce linguistiche riconducibili alla Toscana occidentale confermerebbero, per i materiali che poi confluiranno in $\mathrm{U}$, un passaggio da queste regioni, sicché si sarebbe individuato «uno dei canali attraverso i quali la poesia dei trovatori può essere penetrata in Toscana nel corso del Duecento» (ibid.).

5 Il volume è corredato da una tavola sinottica di confronto tra l'ordine dei componimenti e delle sezioni d'autore in $\mathrm{U}$ e negli altri canzonieri trobadorici, un apparato iconografico e un $c d$-rom, in allegato, che offre la schedatura completa dei 
componimenti e la trascrizione diplomatica del manoscritto. Completano il volume la bibliografia e l'indice dei nomi e delle opere anonime. 